\title{
Article
}

\section{Using bonsai as nature assisted art psychotherapy}

\section{Caroll Hermann*}

1 University of Zululand; hermannc@unizulu.ac.za

* Correspondence: hermannc@unizulu.ac.za; Tel.: +27 825677583

* ORCID ID: 0000-0002-7224-8297

\begin{abstract}
Background: Bonsai integrates relaxation, leisure and art therapy into one medium that can have personal, emotional and commercial value and have benefits beyond the therapist's couch. The art of bonsai, when used as a therapy tool may affect the capacity of the bonsai practitioner to adjust to negative and stressful life events. Objective: The study was initiated to evaluate the benefit of bonsai on the wellbeing of practitioners of the art. Method: 255 bonsai artists participated in a study that examined the effect of bonsai on their well-being. Semi-structured interviews were conducted with bonsai artists. Results: The majority of participants agreed that being able to work on their bonsais affected their moods positively. Discussion: Although bonsai artists did not report better mental health, participants reported an improvement in their mood when working on a bonsai tree. These findings are significant in that it can be especially useful in settings such as psychiatric hospitals, old age homes, orphanages, prisons, etc., as a preventative measure as well as restorative measures in the well-being of patients.
\end{abstract}

Keywords: bonsai, art, psychotherapy, mental health; well-being 


\section{Introduction}

Creative processes are powerful mechanisms for the release of inner emotions [1]. Together with Jungian principles such as self-awareness, transformation, and actualisation, as well as employing rituals, may have the benefit of purging the client from pain or painful events whilst working on a bonsai. It is maintained that art not only contributes significantly to analytical psychology but also manifests as personal expression or form of something more universal that exists within each and every person [2].

Penzes, van Hooren, Dokter, Smeijsters \& Hutschemaekers, [3] state that the art product (and this case, the bonsai) can be seen as an object of transference and therefore can be seen as a representation of the unconsciousness and psychological conflicts of a person. The art object then becomes of value to the client and the therapist.

Psychotherapy is an interpersonal intervention by trained therapists to aid in serious challenges in daily activities [4]. Gardening (being in nature) has always been hailed as a great relaxer and beneficial to mental health. Wolff and Flora [5] state that spending time in nature help improve mental weariness by soothing and renovating the mind. Being active outdoors (gardening) can help to alleviate symptoms of Alzheimer's, dementia, stress, and depression, and improve cognitive function [5].

Being in contact with nature can, for instance, help children to mature cognitively and emotionally, [6] relieve stress in children and adults, connections to families and loved ones. Thus, introducing children to bonsai at an early age, there by simulating these experiences in nature (with a bonsai) could be important opportunities for boosting imagination (dedicating or making a bonsai) and creativity (styling \& designing), cognitive (growing and developing a tree in a small pot) and intellectual development, and social relationships [5].

\section{Psychodynamic Art Therapy}

Art therapy has been well established in psychology as a useful intervention method. Bonsai can serve as a transitional (artful) object as part of the psychoanalytical process. Psychoanalysts regard the relationship between the artist and the objects being created 
objects as inseparable from the concentrated mental energy. This inseparable relationship that stays with the artist could be further explained if the subjective idea or origin of the created object (i.e. bonsai) was obtained [6].

It is in this creative state and the objects that is associated with it (and be remembered as an episode in the artist's life) that more impersonal objects such as the bonsai tree can become representative of the artist's life [6]. Much of Weismann's [7] work refers to Winnicot's multipurpose value of "potential space" when referring to the transitional area (in this instance, the artist using the bonsai to project feelings, etc.) as "that which lies between fantasy and reality" [7] (p1). With regard to this study, the type of potential space includes the play space or the "Bonsai-en" (the bonsai garden), the area of the transitional object refers to the tree and the pot and phenomena, the analytic space, the area of the cultural experience, and the area of creativity refers to processes within the artist.

\section{Bonsai}

Art forms, (such as bonsai) encompasses all that is in nature [8] and the artist can "be" in nature in his own space with the philosophy (f "no kokoro or the spirit of bonsai") described as a combination of the affection for bonsai and enlightenment through nature [7, 8]. During the process of the envisioned idea (of the bonsai), and the artist enters into introspection, and emerges as a state of perfect calm and inner silence, then creativity can take place. Assagioli [9] claims that the significance of challenges, become clearer during this state which he is characterised by a perception of light or enlightenment on problems and situations, which, when they are then understood; their significance will be revealed. When viewing the created object, peace is experienced as self-determined and joy and happiness, harmony, beauty, and power can be felt.

\section{Materials and Methods}


Focus groups were invited to complete online questionnaires and to answer the question:

“What does bonsai mean to you?" Finally, the correspondence was analysed using a thematic analysis approach.

\section{Instruments used:}

Participants were required to complete an online questionnaire based on demographics and their general interest in bonsai, designed by the researcher, as well as a discussion around the question: "What does bonsai mean to you?"

\section{Sample:}

255 international bonsai artists (201 males and 54 females, ranging between the ages of 25 and 83) participated in the study via online questionnaires and/or Skype or email conversations in unstructured interviews with the researcher, which included personal narratives and existing autobiographies made available to the researcher.

\section{Data Analysis:}

Themes were drawn from the narratives and interviews using a thematic analysis approach. During analysis codes were used and themes were established using a codebook. Segments of texts were tagged and segments with similar content were sorted into separate categories for final refinement which were them arranged into major themes [10].

\section{Ethical considerations:}

Permission was obtained from the Research Ethical Committee of the University of Zululand, (UZREC 171110-030-RA Dept.2015/85) as well as informed consent from all participants. Confidentiality and anonymity were ensured by encoding all material. Participants were informed that they could withdraw at any stage. All research protocol was observed.

\section{Results}

The demographic results indicated that 201 participants were male and 54 females although a relatively equal amount of male and females were invited to participate. A small number 
of respondents, $(0,8 \%)$ were below the age of 25 , and $37,3 \%$ between 26 and 40 . The biggest proportion of participant fell in the 41 to 60 age gap and $9 \%$ over the age of 61 [11]. The majority of participants, $(98,8 \%)$ responded that being able to work with their bonsai had a positive and calming effect on them and 95, 7\% declared that not being able to work on their trees, for whatever reason, had a negative effect on their mood [11]. The one $(1,2 \%)$ participant who reported that working on bonsai was not relaxing, was a bonsai vendor and therefore made a living out of selling bonsai.

Subjective correlative analysis of content refers to the data collected during interviews. It was transcribed, however, all other written communications (either email correspondence or participant CV's) were organised in an encodable MS Word document format. This method can be further enhanced by using of codes or the establishment of themes using a codebook; tagging segments of text and sorting segments with similar content into separate categories for final refinement into major themes. The processes above can be described as a "template approach" as it comprises applying similar types of words based on prior research and theoretical perspectives [12].

"Thematic analysis", according to Braun and Clarke [13], involves a process of classification, analysing, and reporting detailed word patterns and/or rich word themes set in the data that was collected. The focus of this analysis was to identify and interpret several aspects of the research topic.

Although thematic analysis is frequently used during research, Braun and Clarke [13] stated that researchers should envisage it as a foundational tool due to its diversity and complex nature. The benefit of using thematic analysis, is its flexibility. Braun and Clarke [13] asserted that analysis becomes exciting when themes are discovered and notions are found to be rooted in the interviews.

Thematic analysis is different from other analytical approaches that attempt to describe commonalities across qualitative data such as "thematic" discourse analysis, or thematic decomposition scrutiny, and grounded theory. Thematic analysis attempts to find patterns in the data, but are contextually bound to and grounded theory. 
Thematic analysis, together with grounded theory form the basis of this research. Grounded theory loosely revolves around generating or discovering a theory from data that was obtained from social research [15].

In this study, thematic analysis was carried out in an "essentialist" manner, which was used to report the experiences of the participants, how those experiences were significant as well as the reality expressed by the participants'. Braun and Clarke [13] stated that it could also be used as a "contextualist" method, utilising the two extremes of "essentialism and constructionism" (p.81). Braun \& Clarke, [13] therefore recognises the ways in which individuals make sense of their experiences and how the social situation imposes on those meanings. Consequently, thematic analysis is a process in which summative objective reality is revealed whilst the surface of the participant's realities is being "unpicked or unraveled" [14] (p.81).

A theme captures an important idea around the data, relative to the research question and embodies some level of recognisable response or significance within the data set. It would be up to the researcher's judgment to select the different patterns or themes and decide on the occurrence in terms of space within each data item and data set [13]. A thematic network is developed by starting from "Basic Themes" working ultimately towards a "Global Theme".

Basic themes form part of the lower order of themes resultant from the qualitative data [16]. Statements are attached to a principal concept and eventually contribute toward the meaning of the overall theme. The "Global Theme" of this study is the artist's impression of "Bonsai" in his or her life.

According to Attride-Stirling [16] and Fereday \& Muir-Cochrane, [14] there are three stages of analysing themes: Stage 1 is the analytical stage where coding of the material takes place, themes are identified and thematic networks are constructed. During Stage 2, theme networks are explored, described and summarised. The final stage of thematic analysis is the integration of the explored patterns are interpreted.

During stage 1 , codes were devised along the lines of Wilber's $[17,11]$ quadrants to organise 
the similar or related segments of text to assist in interpretation. Attride-Stirling [16] advocates strongly for devising such a framework for the credibility of the study.

During Stage 2, the recorded and collected transcripts were screened for abstract themes from the coded text segments. Thematic networks were constructed and finally, the templates of codes and additional coding were applied.

\subsection{Thematic Analysis}

3.1.1. Theme 1 (I Reflection) - Bonsai is irrefutably connected to a love of all things in nature [11] (p.105 - 107).

- Power: All the participants stated that they enjoy the feeling of control which they have over the tree when styling and developing. The more successful they are in keeping the trees alive, the more powerful they feel.

"To make a bonsai trunk taper and the lower branch thicker, while the tendency of a plant is to give most of the nutrients to the top, means that control of the tendency is really necessary in order to achieve a good result. We have to prune the top section of the tree regularly so as to force the nutrient to the lower part. It is an example of controlling the natural tendency in order to achieve a good result." [11, p.105]

- Harmony and order: Bonsai rules, traditions and rituals: All participants acknowledged the need for styling rules, although a small number, representing 5, $9 \%$ (in the quantitative survey), stated that they had never heard of these rules and therefore did not follow them. It was evident in the qualitative survey that the participants who were interviewed and did not follow rules also did not belong to any of the formal bonsai clubs and were therefore not exposed to the strict regimen proclaimed by them. A large proportion of the participants did not follow rules per se but agreed that you "needed to know them, in order to break them", and thereby be considered a true artist. "You have to know the rules, to break them."

People claimed to have some sort of tradition or ritual, which they follow when working on their tree or walking through their bonsai-en. "I just concentrated on my 
tree of choice for a long time. I channeled my imagination toward attempting to design it. When the idea came to me, I started to work."

"I like to wear my happi (traditional Japanese coat worn during festivals). I feel that it focuses me." "I sit down and contemplate what I am about to do, while smoking my pipe, sometimes for hours without doing a thing."

"I have a certain routine; starting by cleaning first the soil surface then the pot and then the trunk and branches. Only after all this do I take a relaxed look at the tree and decide how to proceed and whether or not to change anything. To begin working without the cleaning ritual makes me feel uncomfortable because I could possibly overlook any number of important things."

- Respect for nature and conservation: A true appreciation of art and nature cannot be experienced in isolation. Respect for nature is first and foremost in everyone's mind. "...it has made me more aware of the cultural needs of the flora I see around me, and it has helped me learn to think as a steward of nature rather than a simple user."

All subjects felt that, by planting trees and seeds and by cultivating seeds, they were contributing towards conservation and thereby decreasing the negative impact on Earth (Gaia). "With regard to ecological wellbeing, growing bonsai has made me more aware of the necessity to maintain the growth of trees, jungles and our surroundings. A cut tree will look ugly in our eyes. An old ancient tree will look beautiful and has to be maintained to make it live longer. Growing bonsai has made me love nature more."

"Working with bonsai makes us aware of nature and I think that all bonsai growers are extremely aware of the ecological well-being of nature as a whole. One looks at nature with new eyes and tries to protect our natural heritage."

3.1.2. Theme 2 (Reflection) [11, p.108 - 110] - A deep-seated spirituality is involved in practicing the art. 
- Pleasure: Participants expressed feelings of euphoria when spending time with their trees. These subjective feelings of pleasure were all expressed as "happy" and "peaceful moments", "far better than spending time in social bonsai gatherings".

"Bonsai gives me a creative outlet, helps me relax, helps me focus, and sometimes gives a temporary refuge from conflict."

"...this intriguing activity which is a blend of art and horticulture."

"I don't like people, I like trees, where I find my peace..."

- Contentment: When participants admired their handiwork, they expressed a feeling of contentment, whether the end product was acceptable to other viewers, of a high standard, show quality or not. It was not important for the artist to be admired by his or her peers as the contentment is derived from private viewing and the pleasure obtained from the object. "...the artistic significance of bonsai creation makes me delighted to have this opportunity to display a small number of our better examples in this gallery setting. I hope that you enjoy looking at these artistic forms which never stop growing and so, in that sense, are never finished works of art."

"One always tries to design a tree so as to elicit a reaction from the viewer." "I don't look for my own personality in my creations yet other people have commented on recognising repeated / consistent factors in the trees which reflect my personality and that is important"

- Inner Spaces and Shadows: As with any art, the use of space and shadows is very important for bonsai. One of the traditions of bonsai is, to leave a space for birds to fly through your tree, literally and figuratively. Some artists also leave space for Buddha to sit under the tree.

"During that empty time, I got a vision on planting a small banyan tree." “...It brought joy to my empty time..." 
"Negative spaces, which can also be the "empty space" between branches or foliage, are also shaped and proportioned to appear in balance....see completely through the tree's negative spaces to the background."

Many feel that being able to spend time with your bonsai, is a divine experience in which every decision to cut back, nip or leave to develop is a reflection of the artist's spirit and personality.

"Negative space resonates within my soul to a certain degree but I focus more on the general 'feel' of the tree than on the individual components (as with life)."

"I find this to be key, as I need silence in which to 'lose myself' when working on my trees."

"Negative space is very important for correct balance. As in life, you need the bad to place the good in perspective."

- Silence: Once the artist starts to work on the tree, be it his own, or a demonstration tree, silence is of the utmost importance.

"Silence is very important; I even chase my seven-year-old away."

"Silence allows communion with my trees and results in better concentration."

"Yes, I valued the silence, especially when I was still working. It was wonderful to return from work and concentrate on my trees with no interruptions or other people who needed attention. Very relaxing."

- Spirit guide: "When creating a tree to imitate a tree in nature, one becomes aware"; "Growing bonsai is really a good means by which to train one's spirit." "A small pot and minimal nutrient in the whole life is a good method of selfdiscipline and control of our desire. Consistency in controlling desire is a means to attaining a higher spiritual level."

“...The bonsai was formerly just a natural plant growing wild as a friend of snakes, insects and other low-level animals. Now it (the bonsai) is trained to be a civilised creature. It has to have a tight diet like a monk. It becomes the friend of a human 
being, the highest creature in the world. All of this will enable the tree to become holier in the sense of its spirituality, which will, in turn, allow it to become a higher creature in its next life; an animal or even a human being. The bonsai artist will also advance to a better level due to the discipline and his or her love shown to the trees...."

"This attention and tedious work carries me to a state where I just do it and forget about the rest of the world. I let my thoughts roam while I do the work. I often forget about time and space. This is called meditating. While being part of Zen Buddhism it can very well be done by an ordinary European without any religious or cultural connection with Asia."

3.1.3. Theme 3 (We Reflection) [11, p.110 - 112] - A balance needs to be maintained between what the bonsaist feels toward the tree and the innate need to create.

- Competence: "Having to concentrate when shaping or styling a tree, or keep your mind active when handling a problem tree helps one to stay mentally alert and sharp." "Object lessons found in bonsai, reflect, to a certain extent, God's dealings with us. For example, sometimes I'll remove a perfectly healthy and visually attractive branch from a tree, simply because it doesn't conform with my design for that tree because I'm the artist. God is a much better Artist than I am, and His designs are exactly right. Remembering that makes trust that much easier in difficult situations."

- Respect: "But now I surrender more to God."

"I let the tree tell me what the best shape is."

"I like to be quiet and listen for the voice of God".

"I respect nature as it reflects God's face"

- Connected to life cycles: "A tree also has a soul. A well-styled, healthy bonsai tends to get a higher degree in the next reincarnation. It is already a civilised creature, not 
a wild one. In bonsai training, we also condition ourselves to be more disciplined and to share our love of the bonsai.

Enjoying the bonsai experience (As long as we are not merely motivated by ambition or a desire to be number one) will help us to better ourselves in many ways."

"In my past life, my soul was in a baobab. My soul still lives in a Baobab, even today".

- Meaning of bonsai: "A connection to the real world, to changing seasons, to life, to death, and to natural processes."

- Reflection: "Yes, a desire to reduce our pain or to direct our concentration away from a painful thing is normal."

"Bonsai is like a faithful second wife to me. I can share my love with it. It keeps me busy. I would be sad if anything happened to it. It is really loyal and would never betray me. In the 'Whole life,' it accompanies me. What is more the first wife will never be jealous of the second one."

"When I first saw bonsai I was quite intrigued. I felt that it brought many things together for me. It was very challenging gardening and had to do with scientific botany.

It was serious art, very complex and seemed really impossible for a person from my culture. Well, really impossible things have always challenged me, so I started bonsai and have not stopped since."

"I have developed coping mechanisms for when a tree dies. When one has a thousand trees and does risky things with them then there will be many fatalities. If it broke my heart every time a fatality occurred, then my heart would be broken permanently. I feel like a doctor in a major hospital. I do my best but if a patient happens to die I put it down to experience and forget about it. In theory, this is 
sound practice but it is not always how it works. When a major tree dies it really hurts me deeply."

"Part of my personality is inevitably reflected in my trees."

"One can but wonder if we are philosophical enough in our culture to really reflect what is in our trees. I think that we are still too bound by rules and guidelines to allow more of our personal qualities to come through in our creations, which is a pity."

3.1.4. Theme 4 (Its Reflection) [11, p.110 - 114]- There is a lack of social affinity amongst artists

- Belonging: "It gave me fame, a lot of friends and richness in art appreciation, but I don't need clubs".

"It was a big breakthrough, artistically and also financially, to be accepted to exhibit at BMW in Germany. It was the first time that I received a rather large sum of money for exhibiting trees. It also helped my standing in the bonsai community a lot."

- Social component: "Growing bonsai engenders a certain degree of love for the tree and pride in the result of our creations. On a more mercenary level, the trees grow in value as we work, our success brings recognition as artists and our standing in the organisation is enhanced. Understanding and coping with the interpersonal and political aspects of the foregoing requires tact, diplomacy, and social expertise."

"Although I am not a member of a club I find joy in tending to my own trees and have shown my collection to but a few people."

"I don't belong to a club - Bonsai is my hobby".

"I do not really attend bonsai meetings."

"My locus of control is not in the club." 
- Resources: “...Bonsai has helped me to evolve into a tougher person, more durable in changing situations. I have acquired enough patience to accept the fact that it takes years to create a fine bonsai. Working with bonsai instills in us the discipline, diligence and consistency required to take care of our plants for the rest of our lives."

- “. weeding my bonsai pots, helps me to relax.

- Personal gain: "Growing bonsai makes me aware of the need to care about my personal health and wellbeing." To soothe my soul and reconnect me with people and places long ago. It is where I go for better or for worse, in sickness and in health."

“...is giving me a little more confidence in dealing with people. That's especially significant because, as I may or may not have mentioned to you, I've had a life-long speech impediment."

- Social gain: "Bonsai brings one together with many people all over the world and so broadens one's social spectrum. Once they have met, bonsai growers become friends forever. I believe that a bonsai stranger is just a friend one has not yet met." "Growing bonsai has made me a member of a large society. I have many friends all over the world. It made me famous as well. Anyhow, getting along with many bonsai friends in (the bonsai) society also educated me in the world of politics." "I think it provides a sense of being part of a community. This community includes people from all over the world, including yourself, whom I've never met in the flesh but have come to regard as friends."

“.... Very, but I also like working with a good friend."

- Physical health: "People who grow bonsai don't really need to go to the gym on a daily basis. They get enough exercise by moving their trees around, digging up those growing in the ground, going on digs, mixing soil and re-potting their trees regularly. So one can have enough exercise while working with bonsai." 
"It helped me to overcome major (spinal) trauma in a positive way".

\section{Discussion}

The purpose of this study was to determine whether the art of bonsai has an effect on the mental well-being of people who own bonsai trees. The study provided validation that working, styling, designing and caring for bonsai and other activities in and around bonsai, such as trimming, weeding, watering might have an impact on the capacity of a person to adjust to negative life events.

More explicitly, it was an attempt to determine whether or not being engaged in bonsai leisure activities could have direct and indirect influences on mood and to what extent, if at all, such influences might be relevant to engagement in bonsai activities specifically. Results implied that bonsai activities did have a direct effect on mood adjustment, while at the same time creating a platform for spirituality. All participants, bar one, declared that bonsai activities contributed greatly to the mental wellbeing of individuals. The one person who felt that bonsai did not contribute to mood or relaxing, made a living out of selling bonsai and was therefore not a hobby.

"It's not the stuff of Bonsai that keeps us going it's the spirit of it."; and "It helped me to overcome major trauma in a positive way". All participants agreed that Bonsai helped to make them "feel better".

This study revealed more than the original question and indicated answers regarding the implications of the art on the social, political, cultural and personal ramifications of being involved in the art of bonsai.

The incessant rhythmic cycles represented in nature and in a life being fulfilled through the nourishing of a living thing, provide a form of achievement which caused "spiritual movement" [11, p.152]. Baran, [18] even questions further that bonsai can represent alternative versions of the self and is representative of the artist's stature in the world, (i.e. upright vs. windswept, formal vs. informal, silhouette vs. evergreen, etc.) or suiseki (viewing stones) as representative of the world (dark and mysterious vs. precisely defined 
mountain or hut, solid vs. spirit-opened, etc.).

Lastly, it is not the impact which the artists have had on the bonsai which is important, but rather what the bonsai meant to them personally.

Loy, Dattilo and Kleiber [19] proposed that engaging in leisure activities often provides a degree of stability which is soothing for individuals who have experienced an interruption of their daily activities by negative or disabling life events. However, additional research is required to better define the meaning of the "continuity of leisure engagement and diversity of leisure" range of activities in the lives of persons living with disabilities. Loy, Datillo and Kleiber's [19] study provides further evidence of the importance of remaining actively engaged in life after a negative life event.

The stories and experiences communicated by the bonsai artists in this research have illustrated how bonsai can provide the context for healing, growth, power, gratification, and transformation. It may have demonstrated that artistic expression, caring and tending to a tree can be healing and therapeutic. Further research could investigate the possible benefits of healthy self- expression within artistic contexts on body image, self-confidence, and selfesteem with regards to shapes and styles. It may be of use to the psychology profession to be aware of the possible benefits of nature-assisted therapies, with specific reference to bonsai.

Psychoneuroimmunologists (the interaction between psychological processes and the nervous and immune systems of the human body) describe the relationship between psychosocial and biological aspects in the etiology and development of a disease. The way in which an individual understands and responds to the environment governs both the effect which stress has on the body and the manner in which health behaviors influence and contribute to the neuroendocrine and immune response. Health (and healthy) psychological interventions are intended to control the stress response and enhance health behaviours by coaching individuals on more adaptive methods of deciphering life challenges and encouraging more effective coping responses [20].

Bonsai, along other art therapies can be applied in many settings, such as hospitals, old age homes with minimum disruption and not too much aftercare. 
The narratives by the participants indicated that being involved in the art of bonsai can have many advantages, from a peaceful and relaxing mood to mindfulness to coping mechanisms during physical illness, depression, and bereavement.

\section{Conclusions}

The study was limited to serious bonsai growers and did not include bonsai collectors and bonsai appreciators. This, however, opens the scope for further studies. While results of this study suggested that being engaged in leisure activities has both direct and indirect influences on individuals, there are several limitations associated with the study that limit generalisation and explanation of the findings. Further study is recommended on the direct effect of bonsai as a therapy tool together with clear randomised controlled research, with experimental groups and pre-and post-test assessments.

Other considerations for future research include the type of influence bonsai or nature-based therapies could have on mood or anxiety adjustment. Although results of this study suggested that engagement in bonsai activities had a statistically significant influence on the coping methods of the participants, the study did not disclose the extent to which respondents may have participated in leisure activities more frequently since they were well-adjusted, which highlights the lack of pre-test data. The complexity of the relationship which bonsai activities have on a reciprocal connotation with adjustment requires statistical procedures that were outside the scope of this research. However, further research is needed to examine the possibility that the bonsai model is actually non-recursive or reciprocal. 
Funding: This research received no external funding.

Conflicts of Interest: The author declares no known conflict of interest.

\section{References}

1. Stylianou, N. \& Havran, H. (1998) Thinking about therapy. London: Penguin Group.

2. Whitmont, E., \& Kaufmann, Y. (1973). Analytical Psychotherapy. In R. Corsini, Current Psychotherapies (pp. 85 - 112). Itasca: F.E. Peacock Publishers.

3. Penzes, I., van Hooren, S., Dokter, D., Smeijsters, H. \& Hutschemaekers, G. (2014) Material interaction in art therapy assessment. The Arts in Psychotherapy, http://dx.doi.org/10.1016/j.aip.2014.08.003. Accessed on 7 October 2014.

4. Whitfield, G., \& Williams, C. (2003). The evidence base for cognitive-behavioural therapy in depression: delivery in a busy clinical setting. Advances in Psychiatric Treatment, 9, 21 - 30.

5. Wolff, K., \& Flora, K. (2010). Mental Health and Function - A Literature Review. Retrieved January 12, 2014, from Green Cities: Good Health: http://depts.washington.edu/hhwb/Thm_Mental.html

6. APM (2006) 'Body of knowledge', Educational theory.

7. Weismann, P. (1971). The artist and his objects. International Journal of PsychoAnalysis, 52(4), 401 - 406, accessed on 9 September 2018 at http://eds.b.ebscohost.com/eds/detail/detail?vid=0\&sid=e7bcdc91-a4bb-481c-8bcbf207dd3803b9\%40pdc-v-

sessmgr04\&bdata $=J k F 1 d G h U e X B 1 P X N z b y Z z a X R 1 P W V k c y 1$ saXZ1\#AN=edselc.2$\underline{52.0-0015174907 \& d b=\text { edselc. }}$.

8. Brody, S. (2001). Paul Klee: Art, potential space and the transitional process. Psychoanalytical Review, 88 (3), pp. 369 - 393.

9. Kato, S. (1983). Bonsai no Kokoro: The spirit and philosophy of Bonsai. (D. Fukumoto, Ed., \& D. Fukumoto, Trans.) Retrieved February 12, 2013, from FukuBonsai: www.fukuBonsai.com/5a2.html.

10. Assagioli, R. (1974). Psychosynthesis: height psychology discovering the self and the Self. In P. de Coster, Some Collected Works of Dr. Roberto Assagioli, M.D. (pp. 36 - 49). Gent, Belgium: Satsang Press. 
11. Hermann, C. (2015). Integral ecopsychological investigation of bonsai principles, meaning, and healing. Unpublished Thesis. University of Zululand, South Africa. $\begin{array}{lllll}\text { Accessed } & \text { on } & 25 & \text { June } & 2019\end{array}$ http://uzspace.unizulu.ac.za:8080/xmlui/bitstream/handle/10530/1388/Integral\%20 ecopsychological\%20investigation\%20of\%20Bonsai\%20principles\%2c\%20meanin $\mathrm{g} \% 20$ and $\% 20$ healing.pdf? sequence $=1 \&$ is Allowed $=\mathrm{y}$.

12. DiCicco-Bloom, B.; Crabtree, B. F. The Qualitative Research Interview. Med. Educ. 2006, 40 (4), 314-321. https://doi.org/10.1111/j.1365-2929.2006.02418.x

13. Braun, V., \& Clarke, V. (2006). Using thematic analysis in psychology. Qualitative Research in Psychology, 3 (2), 77 - 101.

14. Fereday, J., \& Muir-Chochrane, E. (2006). Demonstrating rigor using thematic analysis: a hybrid approach to inductive and deductive coding and theme developement. International Journal of Qualitative Methods, 5 (1), 1.

15. Glaser, B., \& Strauss, A. (1967). The discovery of grounded theory: strategies for Qualitative Research. Chicago: Aldine Publishing Company.

16. 2001Attride-Stirling, J. (2001). Thematic networks: An analytic tool for qualitative research. Qualitative Research, 1(3), 385-405.

17. Paulsen, D. (2007). Wilber's integral philosophy: a summary and critique. Journal of Humanistic Psychology, 48 (3), 364 - 388.

18. Baran, R. (2010). Some of what we don't know about the history of magical miniature landscapes and anomalies. Retrieved September 2, 2013, from Phoenix Bonsai: www.phoenixBonsai.com/BigPicture/don'tknow3.html.

19. Loy, D., Dattilo, J., \& Kleiber, D. (2003). Exploring the influence of leisure on adjustment: development of the leisure and Spinal Cord Injury Adjustment model. Leisure Sciences, 25, 231 - 255.

20. Lutgendorf, S., \& Costanzo, E. (2003). psychoneuroimmunology and halth psychology: An integrative model. Brain, Behaviour and Immunity, 17 (4), 225 232. 